PRIMERA CITA DEL ENDEMISMO MEXICANO AGAVE DIFFORMIS A. BERGER (AGAVACEAE) FUERA DE SU ÁREA DE DISTRIBUCIÓN NATIVA

\author{
Jordi López-Pujol ${ }^{1,5}$, Daniel Guillot Ortiz ${ }^{2}$, Pedro Nájera Quezada ${ }^{3}$, Neus \\ NuAlart $^{1}$ y Piet VAN DER MeER ${ }^{4}$
}

${ }^{1}$ Institut Botànic de Barcelona (IBB-CSIC-ICUB), Passeig del Migdia s/n, 08038 Barcelona, España.

${ }^{2}$ Universidad de Valencia, Jardín Botánico, C/ Quart 82, 46008 Valencia, España. ${ }^{3}$ Himno Nacional 1907, 78280 San Luis Potosí, México.

${ }^{4}$ Camino Nuevo de Picaña s/n, 46014 Valencia, España.

${ }^{5}$ Autor para correspondencia: jlopez@ibb.csic.es

\title{
RESUMEN
}

La especie endémica mexicana Agave difformis A. Berger (Agavaceae) se reporta por primera vez fuera de su área de distribución nativa. Se ha localizado una pequeña población compuesta por dos núcleos separados por unos $50 \mathrm{~m}$ cerca de la ciudad de Tarragona (Cataluña, España). Se ha realizado su censo (contabilizándose un total de 139 rosetas, 46 de las cuales son adultas), además de un estudio morfométrico. La población, que parece bien establecida y con tendencia a la expansión, se encuentra bajo un pequeño pinar con restos de maquia litoral. La profusión de especies alóctonas (y algunas autóctonas usadas en jardinería) como acompañantes indica que la población de $A$. difformis podría haberse originado a partir del abandono de restos de poda.

Palabras clave: Agave, España, flora alóctona, Tarragona.

\begin{abstract}
The Mexican endemic Agave difformis A. Berger (Agavaceae) is reported for the first time out of its native range. A small population composed of two groups separated by $50 \mathrm{~m}$ has been located near the city of Tarragona (Catalonia, Spain). A census of the population has been carried out (a total of 139 rosettes were counted, of which 46 were regarded as
\end{abstract}


adults) together with a morphometric study. The population of $A$. difformis, which occurs under pine trees in remnants of Mediterranean coastal scrub, appears to be well-established and expanding. The large number of accompanying alien species (as well as some native taxa used as ornamentals) suggests that the population of A. difformis could have originated from dumped garden debris.

Key words: Agave, alien flora, Spain, Tarragona.

\section{EL GÉNERO AGAVE EN LA PENÍNSULA IBÉRICA}

El género Agave L. consta de alrededor de 200 especies distribuidas de forma natural desde Estados Unidos de América hasta Venezuela, además de las islas del Caribe (Good-Avila et al., 2006). La mayoría de sus componentes son nativos de México (alrededor de 75\%), y cerca de $70 \%$ de éstos son endemismos exclusivos de este país norteamericano (Eguiarte et al., 2013). Con importantes usos alimentarios, medicinales e industriales, su aprovechamiento se remonta a hace unos 9000 años como mínimo (Callen, 1965; Gentry, 1982). Los representantes del género son ampliamente cultivados en las regiones tropicales, subtropicales y templadas de todo el planeta, incluyendo la península ibérica, de donde se conoce la presencia de al menos 85 taxones (Guillot y Meer, 2006a; 2011). En esta región europea se han descrito incluso nuevos taxones a partir de material alóctono (p. ej. Agave gonzaloi; Guillot y Meer, 2004b).

El género Agave se registra desde el siglo XVI en España. Charles de l'Écluse (Clusius) nos proporciona la primera noticia sobre un ejemplar (probablemente $A$. americana) cultivado en Valencia, gracias a la información obtenida del médico y profesor valenciano Juan Plaza (Ramón-Laca, 1999). Cuando Clusius la observó en 1564-1565 al parecer ya era común en estas tierras, puesto que era popularmente conocida como "hilo y aguja" por las espinas finales de las hojas y porque las fibras interiores servían como hilos (Boreau, 1850; Guillot y Meer, 2004c). Si exceptuamos el caso de $A$. americana, presente ya en el siglo XVI como se comenta más arriba, numerosas especies se reportan desde el siglo XIX y principios del XX, como por ejemplo A. angustifolia, A. atrovirens, A. fourcroydes y $A$. salmiana (Guillot et al., 2009c). La planta más cultivada es sin duda $A$. americana, muy común en jardines y fincas agrícolas. Su presencia en estado silvestre está ampliamente documentada desde el siglo XVIII (Guillot et al., 2009c) y en la actualidad es una especie muy frecuente en espacios fuertemente humanizados (zonas periurbanas, áreas agrícolas, bordes de carreteras y caminos, etc.). 
En la península ibérica y las islas Baleares han sido citados numerosos representantes del género Agave, la mayoría de origen mexicano o bien con un área de distribución que incluye a este país, junto a otros taxones conocidos solamente en cultivo descritos por primera vez a partir de ejemplares procedentes de jardines europeos. Podemos citar principalmente, como hemos indicado más arriba, a $A$. americana, en concreto su subsespecie americana, que se encuentra en España ampliamente naturalizada por todas las provincias insulares y litorales mediterráneas y suratlánticas así como en Portugal, principalmente cerca de la costa en el centro y sur. También han sido mencionados en estado silvestre en la península y/o las islas Baleares los taxones Agave amaniensis Trel. \& W. Novell, A. americana L. var. marginata Trel., A. americana L. var. medio-picta Trel., A. americana L. var. striata Trel., A. angustifolia Haw. y su var. marginata hort. ex Gentry, A. atrovirens Karw., A. cerulata Trel. subsp. dentiens (Trel.) Gentry, A. decipiens Baker, A. desmetiana Jacobi, A. fourcroydes Lem., A. franzosini Baker, A. funkiana K. Koch \& C. D. Bouché, A. ingens A. Berger y su var. picta (Salm-Dyck) A. Berger, A. karwinskii Zucc., A. lechuguilla Torr., A. lophantha Schiede, A. lurida Aiton, A. salmiana Otto ex Salm-Dyck y su var. ferox (K. Koch) Gentry, A. segurae D. Guillot \& P. Van der Meer, A. sisalana Perrine ex Engelm. y su var. armata Trel., A. weberi J. F. Cels ex J. Poiss., A. $\times$ cavanillesii D. Guillot \& P. Van der Meer y A. $\times$ winteriana A. Berger (Guillot y Meer, 2003a, b, c, 2004a, 2005, 2006b, 2008, 2009, 2010, 2013a, b; Gui1lot, 2006; Serra, 2007; Smith y Figueiredo, 2007; Guillot et al., 2009c; Sánchez et al., 2009; Sanz et al., 2011; Rubal et al., 2013; Guillot y Sáez, 2014; Sáez et al., 2014; Aymerich, 2015). Algunas de ellas (A. americana, A. fourcroydes, A. sisalana) han mostrado un fuerte carácter invasor mediado por su reproducción clonal, especialmente (pero no exclusivamente) en suelos arenosos del sur peninsular (Badano y Pugnaire, 2004; Sanz et al., 2004), y que generalmente se ha traducido en una pérdida de la diversidad de especies nativas (Badano y Pugnaire, 2004). En los últimos años se han llevado a cabo algunas acciones de erradicación, como por ejemplo la retirada manual de individuos de A. americana en el Parque Natural de Calblanque, en Murcia (Sanz et al., 2004).

\section{AGAVE DIFFORMIS, ENDEMISMO MEXICANO RESTRINGIDO}

Uno de los taxones menos conocidos y con una distribución más limitada (Fig. 1) dentro del género es Agave difformis A. Berger. Del grupo Marginatae, se trata de un taxón morfológicamente cercano a A. funkiana y A. lechuguilla que, junto a los 
cuales, forma un complejo taxonómico aún no resuelto (A. García-Mendoza, com. pers.). Agave difformis es localmente abundante en la Barranca de Metztitlán y en las áreas colindantes en el estado de Hidalgo (México), pero fuera de este área es raro (Eguiarte et al., 2013). Gentry (1982) indicó que habita en suelos gruesos calcáreorocosos en la cara desértica de la Sierra Madre Oriental en los estados de San Luis Potosí e Hidalgo, en elevaciones entre 1560 y 1875 m. Arzate (2009), en Metztitlán, la cita en suelos derivados de roca basáltica. Si se consideran solamente las poblaciones de Hidalgo, parece tratarse de un agave muy local, puesto que se encuentra en un área de solamente $400 \mathrm{~km}^{2}$ (Eguiarte et al., 2013). Sin embargo, puede llegar a ser muy abundante en los lugares donde se ha observado; por ejemplo, Eguiarte et al. (2013) estimaron una población de más de tres millones de rosetas sólo para la Barranca de Metztitlán. En los últimos años han aparecido varias referencias que amplían su área conocida de distribución al estado de Querétaro (Arias y Sánchez-
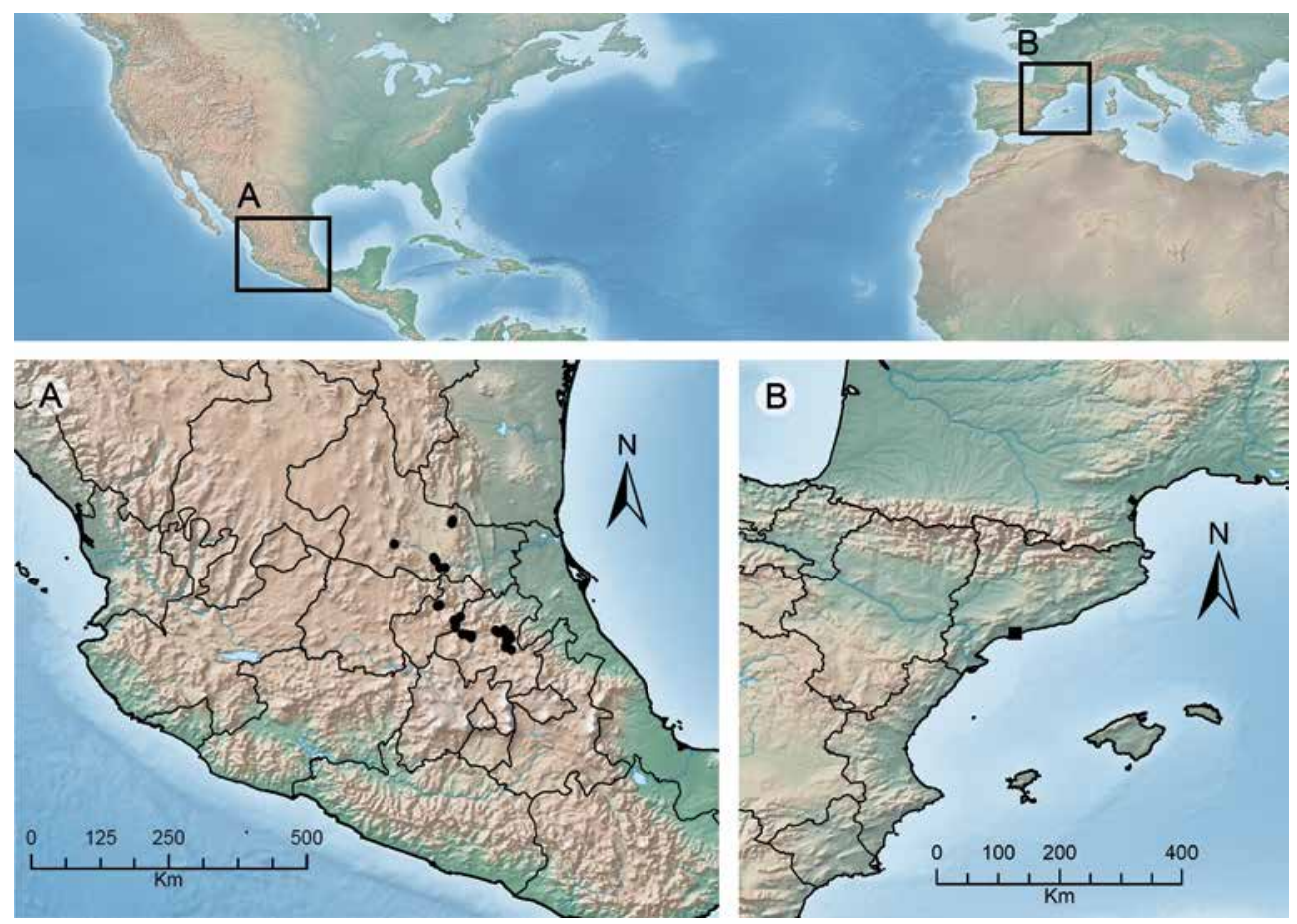

Fig. 1. Localización de las poblaciones naturales de Agave difformis, en su área nativa (México, en los estados de Hidalgo, Querétaro y San Luis Potosí; recuadro A) y fuera de ella (en España, en la comunidad autónoma de Cataluña; recuadro B). 
Martínez, 2010; Zepeda-Martínez et al., 2013; y espécimen depositado en el Herbario Nacional de México (AGA804233), recolectado en el municipio de Peñamiller; http://unibio.unam.mx/collections/specimens/urn/IBUNAM:MEXU:AGA804233).

Agave difformis fue descrita a partir de individuos cultivados, probablemente de origen mexicano (Standley, 1920). Berger (1915) indica que el tipo procede de la planta durante largo tiempo existente en La Mortola (Italia) y otros jardines europeos. Gentry (1982) señala que encontró un número importante de ejemplares de esta especie en macetas en Palermo (Italia) en 1969.

Según Gentry (1982) y Thiede (2001), Agave difformis forma rosetas subcaulescentes, variables, de 70-100 × 100-150 cm. Las hojas son muy polimórficas, rectas, falcadas o sinuosas, de 50-80 $\times$ 4-6 cm, de color verde a amarillo-verde, $\mathrm{y}$ con márgenes rectos $\mathrm{u}$ ondulados, firmes o separables, predominantemente gris claro. Los dientes marginales son también muy variables, generalmente de 5-10 mm, de color marrón oscuro a gris, espaciados $2-3 \mathrm{~cm}$, raramente dobles, en ocasiones con dientes más pequeños entre los otros, o incluso muy reducidos o enteramente ausentes. El nombre dado por Berger ("difformis") se refiere presumiblemente a la presencia o ausencia de dientes, que caracteriza el clon que conoció (Gentry, 1982). La espina terminal es cónico-subulada, de 1.5-3 cm, de color marrón oscuro a gris. Las inflorescencias pueden alcanzar una gran altura $(3.5-5 \mathrm{~m}$; hasta $6 \mathrm{~m}$ según Scheinvar, 2008, en referencia a ejemplares de Metztitlán), espiciformes, con flores en el 1/3-1/2 superior de la inflorescencia. Las flores son pequeñas, de 30-40 mm, con tépalos de color verde claro a amarillo y rosa. Rocha et al. (2005) y Scheinvar (2008) indican que la inflorescencia tiene de promedio 90 flores, y que una planta puede producir aproximadamente 740 flores (de las cuales apenas la mitad acaban produciendo fruto). Según Scheinvar (2008), un fruto puede tener de media 117 óvulos, de los cuales poco más de un tercio forman una semilla viable (que son dispersadas por el viento). En cuanto a la polinización, se trata de una especie generalista (Rocha et al., 2005; Trejo-Salazar et al., 2015). Se han citado como polinizadores a murciélagos nectarívoros (Leptonycteris yerbabuenae, L. nivalis y Choeronycteris mexicana), que son atraídos por la gran cantidad de néctar que esta especie produce durante la noche, así como por abejas y abejorros (particularmente Apis mellifera, pero también Bombus pennsylvanicus sonorus y Lasioglossum (Lasioglossum) sp.) y diversos esfíngidos (Erinnyis ello, Pachylia ficus, Sphinx lugens y Agrius cingulatus, de acuerdo con Trejo-Salazar et al., 2015).

Esta especie presenta niveles de diversidad genética en la media dentro del género Agave $\left(H_{\mathrm{e}}\right.$ (heterocigosis esperada) $=0.205$, obtenida con marcadores ISSR (Inter Simple Sequence Repeats; Eguiarte et al., 2013). La diferenciación genética 
entre sus poblaciones, aunque no muy elevada $\left(F_{\mathrm{ST}}\right.$ (coeficiente de diferenciación genética) = 0.113; Eguiarte et al., 2013), debe calificarse de importante teniendo en cuenta que se trata de un taxón de distribución muy restringida. Según Rocha (2006), presenta una estrecha relación con A. xylonacantha y Agave sp. (una especie todavía por describir, al parecer endémica de la Barranca de Metztitlán) desde el punto de vista genético. Gómez-Pompa (cf. Gentry, 1982) indica que la fibra de xixi (denominación local de $A$. difformis en Ixmiquilpan) era macerada y empleada para jabón.

\section{PRIMERA CITA DE AGAVE DIFFORMIS COMO PLANTA ALÓCTONA}

En el curso de una prospección de flora naturalizada en la provincia de Tarragona (noreste de la península ibérica) se descubrió una pequeña población de Agave difformis. La identificación se realizó mediante literatura especializada (Gentry, 1982; Thiede, 2001) y consulta de los herbarios ARIZ, ASU, DES, K, MEXU, MO, SLPM y US. Se ha depositado un pliego testigo en el herbario del Instituto Botánico de Barcelona (BC) (Fig. 2). También se ha trasplantado uno de los ejemplares que conforman la población al Jardín Botánico de Barcelona para su conservación ex situ. En octubre de 2014 se realizó un primer censo de los ejemplares, que se repitió en agosto de 2015 (diferenciando estadios de desarrollo). Aunque en la visita inicial (2014) se tomaron algunas medidas, en la segunda (2015) se hicieron numerosas de tipo morfométrico de una manera sistematizada que incluyeron: (1) altura de la roseta, (2) anchura de la roseta (diámetro mayor), (3) número de hojas de la roseta, (4) longitud de la hoja, (5) anchura de la hoja (en su punto más ancho, que se encuentra entre el tercio proximal y la mitad de la hoja), (6) longitud de los dientes marginales, (7) separación entre dientes, y (8) longitud de la espina terminal. Para tal propósito se escogieron solamente las rosetas que consideramos como "adultas" (las que tenían como mínimo $0.7 \mathrm{~m}$ de altura). Mientras que las tres primeras medidas (altura y anchura de la roseta más el número de hojas) se hicieron de todas las rosetas adultas, las de la longitud de la hoja, la anchura de la hoja y la longitud de la espina terminal se tomaron de cinco hojas (que nunca fueron basales ni terminales) seleccionadas al azar de diez rosetas adultas también escogidas aleatoriamente. De entre estas cinco hojas se eligió una al azar de la que se midió la longitud de todos los dientes marginales (excepto los situados en el décimo proximal y el tercio distal, que se descartaron) y la distancia entre éstos. Finalmente, se anotaron las especies acompañantes y se tomaron numerosas fotografías (mediante una cámara digital Finepix HS30; Fujifilm, Tokio, Japón). 
López-Pujol et al.: Agave difformis, endemismo mexicano fuera de su área de distribución nativa

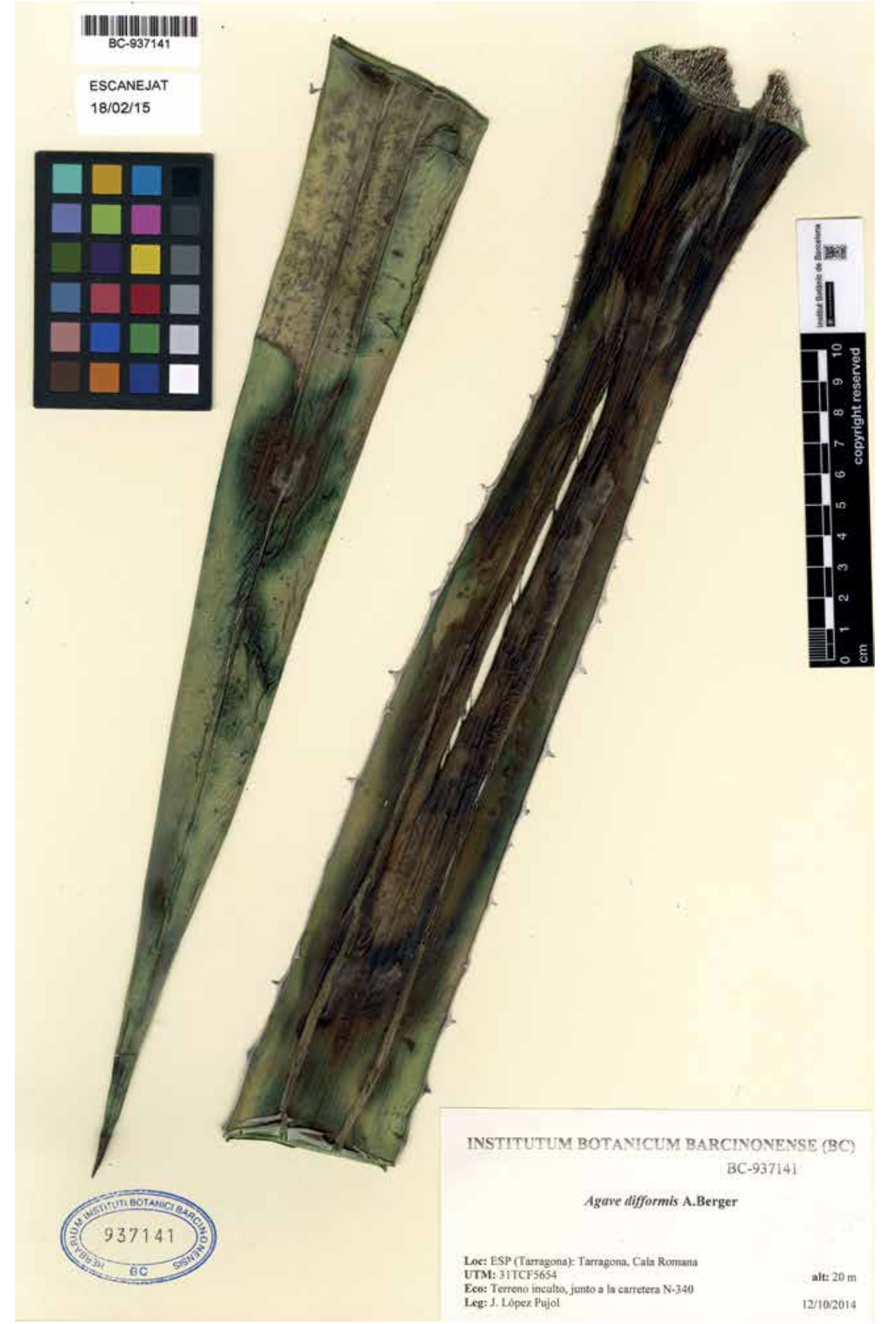

Fig. 2. Hoja de un individuo de Agave difformis de la población de Tarragona (España). Pliego testigo depositado en el herbario del Instituto Botánico de Barcelona (BC 937141). 
Agave difformis A. Berger, Agaven: 95. 1915.

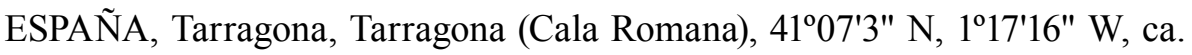
$20 \mathrm{~m}$, terreno inculto, junto a la carretera N-340, 12.X.2014, J. López-Pujol (BC 937141; Fig. 2).

Dicha población consta de dos pequeños núcleos. El primero ocupa una superficie aproximada de $12 \mathrm{~m}^{2}$ (Fig. 3A), con un total de 34 rosetas en 2015 (30 en 2014). El segundo es de mayor tamaño, de unos $45 \mathrm{~m}^{2}$, con 105 rosetas en 2015 (84 en 2014) (Cuadro 1). La población parece, por tanto, estar en expansión, ya que presentó un incremento de $18 \%$ en el número de rosetas en apenas 10 meses. Los dos núcleos están separados por unos escasos $50 \mathrm{~m}$. De los 139 elementos, la mayoría (93) podemos considerarlos como "juveniles" (aquellos con una roseta inferior a 0.7 m; Fig. 3B), y ninguno portaba estructuras reproductivas. De entre los últimos, hay 29 que midieron menos de $30 \mathrm{~cm}$ de altura y que probablemente hayan nacido en la presente temporada (los cuales, por tanto, podemos asimilar a la fase de "hijuelo"). De hecho, la comparación entre los censos por fenofases entre 2014 y 2015 indica que, mientras algunos elementos juveniles (7) habrían alcanzado el estadio de madurez, la mayoría de las rosetas que en 2014 estaban en el estadio de hijuelo ya habrían pasado a ser juveniles 10 meses más tarde (Cuadro 1).

Las 46 rosetas adultas promediaron $91.4 \mathrm{~cm}$ de alto y $133.3 \mathrm{~cm}$ de ancho (Cuadro 2), dentro del intervalo descrito para la especie (véase más arriba). Sin embargo, algunas superaron ligeramente tales dimensiones (la de mayor porte, por ejemplo, era de $110 \mathrm{~cm}$ de altura por $180 \mathrm{~cm}$ de anchura) lo que puede atribuirse a que éstas crecen en un lugar parcialmente sombreado (Fig. 3D). Respecto a las hojas, promediaron $81.4 \mathrm{~cm}$ de largo por $3.8 \mathrm{~cm}$ de ancho (Cuadro 2), aunque en los individuos de mayor porte superaron $95 \mathrm{~cm}$ de longitud y alcanzaron $5 \mathrm{~cm}$ de anchura. Las rosetas adultas en general presentaron pocas hojas (17 de media por roseta; con un máximo de 29). Los dientes (Figs. 2 y 3 C) por lo común fueron más cortos $(2.6 \mathrm{~mm}$ de media, aunque en algunos individuos superaron los $5 \mathrm{~mm}$ ) y ligeramente con menos separación entre ellos $(16.5 \mathrm{~mm})$ respecto al protólogo de la especie. La longitud de las espinas terminales sí se situó dentro de los valores esperados para Agave difformis $(1.54 \mathrm{~cm}$ de promedio; Cuadro 2). En la población no hay restos de inflorescencias ni rosetas secas, lo que parece constatar su origen reciente. Es probable que las 139 rosetas contabilizadas provengan de muy pocas plantas madre, dado que la especie produce largos rizomas de los que emergen hijuelos de reproducción vegetativa (Fig. 3E).

Aun tratándose de un complejo taxonómico cuyas especies son de muy difícil delimitación, tanto las claves de Gentry (1982) como las descripciones de éste y de 
López-Pujol et al.: Agave difformis, endemismo mexicano fuera de su área de distribución nativa
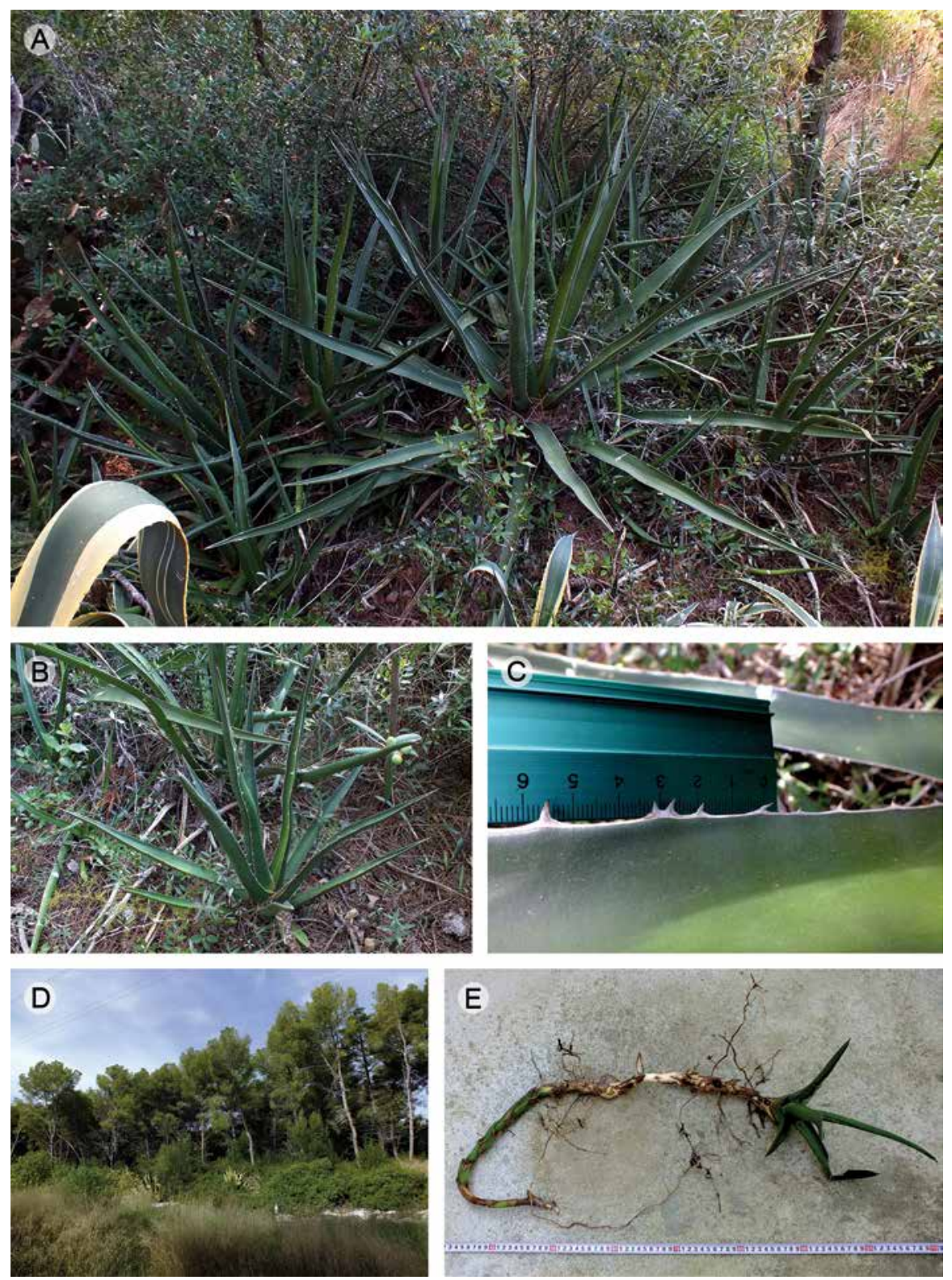

Fig. 3. Agave difformis. A. vista parcial del núcleo pequeño de la población ibérica (en primer plano, el individuo adulto de mayor porte); B. individuo juvenil (altura de la roseta inferior a $0.7 \mathrm{~m}$ ); C. detalle de la separación de los dientes de las hojas en un individuo adulto; D. hábitat que ocupa la población de $A$. difformis (pequeño pinar de Pinus halepensis con restos de maquia litoral); E. detalle del rizoma. Fotografías J. López-Pujol. 
Cuadro 1. Número de rosetas de Agave difformis censadas (y porcentaje) para cada una de las fenofases en octubre de 2014 y agosto de 2015 .

\begin{tabular}{lll}
\hline Estadio & 2014 & 2015 \\
\hline Hijuelo & $38(33.3 \%)$ & $29(20.9 \%)$ \\
Juvenil & $37(32.5 \%)$ & $64(46.0 \%)$ \\
Adulto vegetativo & $39(34.2 \%)$ & $46(33.1 \%)$ \\
Adulto reproductor & $0(0 \%)$ & $0(0 \%)$ \\
\hline
\end{tabular}

Nota: en el censo de 2015 no se ha detectado ningún individuo muerto ni desaparecido respecto al censo de 2014 (a excepción del individuo que se extrajo en 2014 para su conservación ex situ en el Jardín Botánico de Barcelona).

Cuadro 2. Variables morfométricas medidas en la población ibérica de Agave difformis.

\begin{tabular}{lcrc}
\hline \multicolumn{1}{c}{ Variable } & Tamaño muestral & \multicolumn{1}{c}{ Media } & Desviación estándar \\
\hline Altura de la roseta & $N=46$ & $91.35 \mathrm{~cm}$ & $11.67 \mathrm{~cm}$ \\
Anchura de la roseta & $N=46$ & $133.26 \mathrm{~cm}$ & $28.31 \mathrm{~cm}$ \\
Número de hojas de la roseta & $N=46$ & 16.98 & 3.96 \\
Longitud de la hoja & $N=50$ & $81.38 \mathrm{~cm}$ & $7.80 \mathrm{~cm}$ \\
Anchura de la hoja & $N=50$ & $3.83 \mathrm{~cm}$ & $0.44 \mathrm{~cm}$ \\
Longitud de los dientes & $N=425$ & $2.59 \mathrm{~mm}$ & $0.88 \mathrm{~mm}$ \\
$\quad$ marginales & $N=405$ & $16.48 \mathrm{~mm}$ & $8.73 \mathrm{~mm}$ \\
$\begin{array}{l}\text { Separación entre los dientes } \\
\quad \text { marginales }\end{array}$ & $N=50$ & $1.54 \mathrm{~cm}$ & $0.13 \mathrm{~cm}$ \\
$\quad \begin{array}{l}\text { Longitud de la espina } \\
\quad \text { terminal }\end{array}$ & & & \\
\hline
\end{tabular}

Thiede (2001) sitúan las plantas observadas en Tarragona como pertenecientes a Agave difformis. Dichos ejemplares son de mucho mayor porte que los de la especie probablemente más cercana desde el punto de vista morfológico, A. lechuguilla (que suelen medir 30-50 $\times$ 40-60 cm), aunque de tamaño parecido al de otro taxón muy vinculado, A. funkiana (60-90 × 120-180 cm; Gentry, 1982; Thiede 2001). Respecto a las hojas, también son significativamente mayores que las de A. lechuguilla (25-50 $\times$ 2.5-4 cm; Gentry, 1982; Thiede 2001) y carecen de la banda central decolorada propia de A. funkiana (Figs. 3A y 3B). La longitud de los dientes de los ejemplares tarraconenses es, en cambio, más acorde con lo esperable para $A$. lechuguilla (dien- 
tes, 2-5 mm; separación entre los dientes, $1.5-3 \mathrm{~cm}$ ) e incluso A. funkiana (dientes, 3-5 mm; separación entre los dientes, 1-2.5 cm; Gentry, 1982; Thiede 2001). En todo caso, debe tenerse en cuenta el gran polimorfismo de los dientes que se ha descrito en $A$. difformis (éstos pueden estar significativamente reducidos o incluso ausentes; Gentry, 1982); dicha variabilidad puede observarse perfectamente en los pliegos recolectados por H. S. Gentry: hay individuos con dientes casi inexistentes (por ejemplo, en el espécimen Gentry 20095) a otros que alcanzan $10 \mathrm{~mm}$ (p. ej. Gentry 20081). Además, para algunas de las localidades de la especie en México podría existir introgresión de A. lechuguilla en base a lo indicado por Gentry (1982); y así los individuos observados en Tarragona bien podrían corresponder a uno de estos genotipos con "trazas" de A. lechuguilla. Por otra parte, los dientes de las plantas tarraconenses raramente están inclinados hacia abajo, como es característico en $A$. lechuguilla y A. funkiana, y tampoco presentan coloración oscura (Figs. 2 y 3C), como es esperable en A. funkiana (Gentry, 1982; Thiede 2001). Finalmente, como último carácter diagnóstico cabe mencionar la irregularidad en el tamaño de los dientes y la separación entre éstos en $A$. difformis (característica observada en las rosetas de Cataluña; Figs. 2 y 3 C), en contraposición a lo descrito para $A$. funkiana (Gentry, 1982; Thiede 2001).

La población de Tarragona se encuentra bajo un pequeño pinar de Pinus halepensis (Fig. 3D), con restos de maquia litoral (Asparagus acutifolius, Ceratonia siliqua, Chamaerops humilis, Olea europaea var. sylvestris, Pistacia lentiscus, Rhamnus alaternus, Rhamnus lyciodes, Rubia peregrina y Smilax aspera). Hemos observado numerosos taxones alóctonos, destacando Agave franzosini y A. ingens var. picta (esta última entremezclada con los individuos de A. difformis), Albizia julibrissin, Aloe maculata, Araujia sericifera, Asparagus plumosus y A. sprengeri, Cotoneaster pannosus, Iris germanica, Lantana camara, Ligustrum lucidum, Mirabilis jalapa, Opuntia sp. (muy abundante), Opuntia cf. ficus-indica, Pittosporum tobira, Plumbago auriculata y Senecio angulatus. La presencia de estos xenófitos, la mayoría de ellos ampliamente usados en jardinería, junto con Nerium oleander y Coronilla cf. glauca (especies, aunque autóctonas, también usadas como ornamentales, especialmente la primera), no resulta sorprendente dada la cercanía de una de las principales -y más antiguas- urbanizaciones de casas unifamiliares de la ciudad de Tarragona (Cala Romana). Los ejemplares de $A$. difformis observados, por consiguiente, es probable que procedan del abandono de restos de poda, tal y como se ha reportado para otros taxones de Agave y otras suculentas en la región (Guillot et al., 2009a, b, c). No descartamos que la especie se cultive de forma relativamente frecuente en jardines de la zona; de hecho, hemos podido observar una pequeña po- 
blación plantada, formada por aproximadamente medio centenar de ejemplares (de los cuales uno presentaba inflorescencia), en una isleta de la red viaria pública de un municipio cercano (Salou, a menos de una veintena de kilómetros de la localidad estudiada de Tarragona; Fig. 4).

Nuestra búsqueda bibliográfica indica que los ejemplares de Tarragona conformarían la primera localidad de Agave difformis fuera de su área nativa en México en estado silvestre. Aunque la ausencia de inflorescencia dificulta su identificación, los caracteres vegetativos sitúan a los individuos observados dentro de $A$. difformis. Con los datos demográficos y ecológicos de que disponemos, podemos clasificar de manera provisional a $A$. difformis como un metáfito hemiagriófito según la terminología propuesta por Kornas (1990) para las especies alóctonas. Agave difformis fue probablemente introducida en la segunda mitad del siglo XX en la península ibérica bajo otro nombre. Comercializada a principios del siglo XXI por Viveros Jadeflor (Elche, provincia de Alicante, España), posteriormente a partir de este material ha sido reproducida y comercializada por Viveros Vangarden (Picanya, provincia de Valencia, España). Recomendamos el seguimiento periódico de esta población para obtener más datos sobre su comportamiento demográfico, además de realizar prospecciones adicionales en áreas cercanas. También creemos conveniente llevar a cabo estudios específicos encaminados a la delimitación filogenética de A. difformis y de los taxones con los que está íntimamente ligado. Hay que tener en cuenta que, además de A. lechuguilla y A. funkiana, algunos autores como M. Greulich (com. pers.) amplían este complejo taxonómico a otras entidades como A. albomarginata y A. lophantha (este último muy relacionado con A. funkiana según Gentry, 1982, y Thiede, 2001).

El hecho de que un taxón con un área de distribución natural muy restringida geográficamente (como es el caso de Agave difformis) se naturalice en otras comarcas, en ocasiones muy remotas, no es un hecho aislado. Durante los últimos años en la España peninsular, especialmente a lo largo de sus zonas costeras mediterráneas, han sido citados numerosas plantas de taxones alóctonos escapados de cultivo que en su región de origen tienen una distribución muy reducida. Muchos de estos casos pertenecen a lo que denominamos comúnmente como "cactus y plantas suculentas", parte de ellos citados por primera vez por los autores de este artículo en España, y en ocasiones, en Europa. Entre las razones para su establecimiento en la España mediterránea probablemente pueda citarse la enorme transformación de este territorio (lo que, como es bien sabido, facilita el establecimiento de especies alóctonas; Mack et al., 2000), un clima cálido y relativamente árido, generalmente libre de heladas, y una importante incidencia en el empleo de plantas introducidas suculentas y crasas 

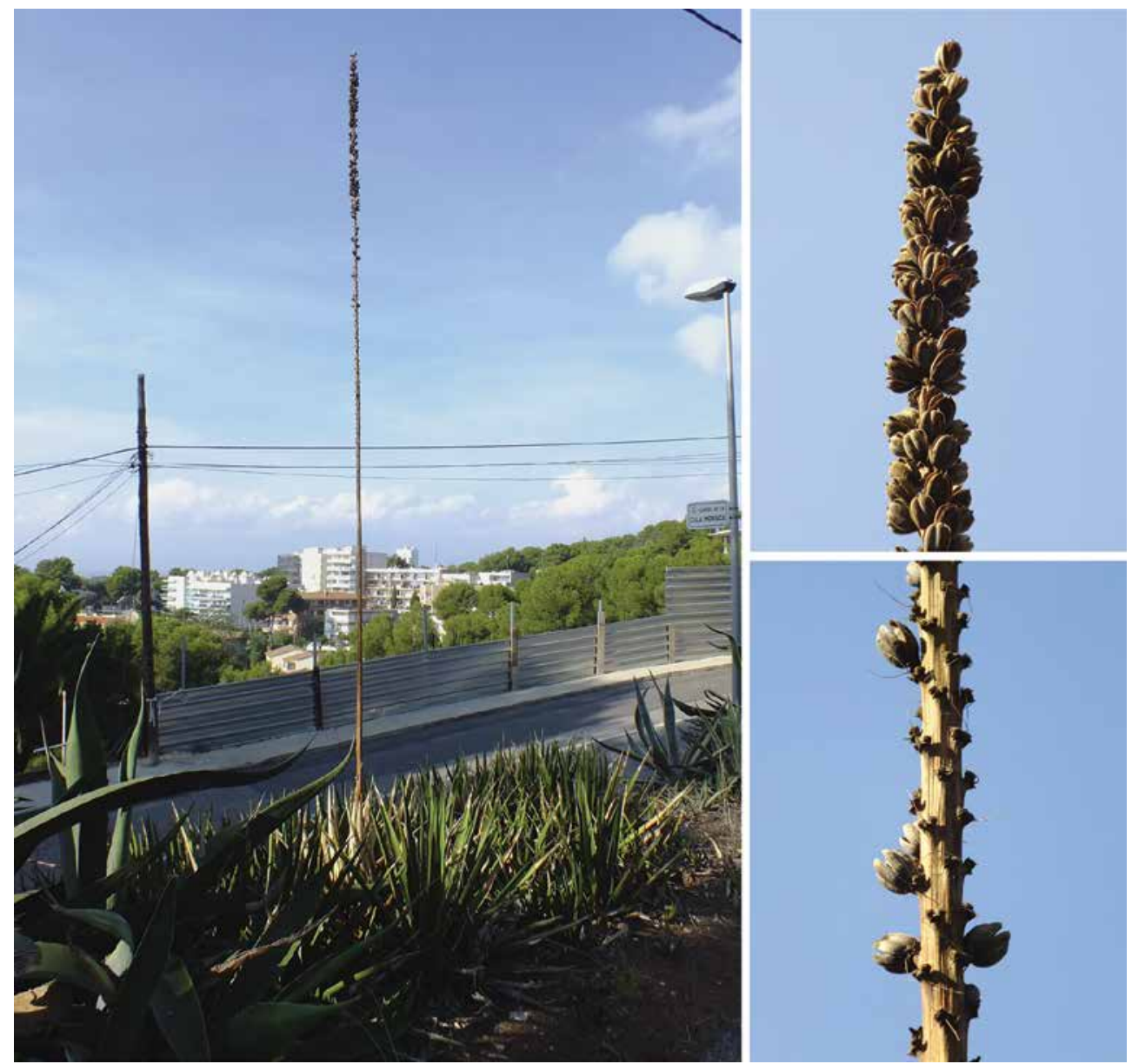

Fig. 4. A la izquierda, vista de los ejemplares plantados en una isleta de la red viaria de Salou. A la derecha, pueden observarse detalles de una inflorescencia (ya seca) de uno de los individuos. Fotografías J. López-Pujol.

en jardinería (agravada por la importante concentración de viviendas de segunda residencia con jardín especialmente en las zonas cercanas a la línea de costa). Dentro del mismo género Agave, podemos destacar A. decipiens (citado por primera vez en la península por Guillot y Meer, 2003a), que crece solamente en zonas costeras de Florida (Gentry, 1982; Thiede, 2001) y Agave lurida (citado por Guillot y Meer, 2008), que es nativa de una pequeña área en las tierras altas del estado mexicano de Oaxaca (Gentry, 1982). Dentro de la familia Cactaceae hay numerosos casos de 
endemismos restringidos que se han naturalizado aquí (véase Guillot et al., 2009b para el detalle de las citas ibéricas): por ejemplo, del género Cylindropuntia ha sido citada Cylindropuntia $\times$ tetracantha, conocida solamente de la región alrededor de Tucson, Arizona (Britton y Rose, 1919); mientras que de Opuntia podríamos destacar la presencia, entre otros, de O. engelmannii var. linguiformis, de la que Anderson (2001) indica que es aparentemente nativa del centro de Texas, Opuntia microdasys var. albispina, observada por Bravo-Hollis (1978) cerca de la ciudad de San Luis Potosí, y Opuntia phaeacantha var. flavispina, también restringida a algunas zonas de Arizona (Earle, 1980). También existen ejemplos de plantas no americanas; dentro de la familia Crassulaceae, se han naturalizado en la península ibérica dos endemismos locales de las islas Canarias del género Aeonium (Guillot et al., 2009a): Aeonium haworthii, que se encuentra restringido al norte de la isla de Tenerife (Nyffeler, 2003) y Aeonium percarneum, de distribución restringida a la isla de Gran Canaria (Nyffeler, 2003). Por último, y también crasulácea, podemos destacar $\mathrm{Ka}$ lanchoe delagoensis (Guillot et al., 2015), especie nativa de la provincia malgache de Toliara y no solo naturalizada en la España peninsular sino en una larga lista de países (Invasive Species Compendium, 2015).

\section{AGRADECIMIENTOS}

Agradecemos al Sr. José Manuel Ramos la ayuda prestada en la medición de las variables morfométricas en la población natural, al Sr. Samuel Pyke por encargarse del cultivo de un ejemplar de Agave difformis en el Jardín Botánico de Barcelona, y a los doctores Luis E. Eguiarte, Abisaí García-Mendoza, Michael Greulich, Alejandro Martínez-Palacios y Joachim Thiede por sus comentarios de índole taxonómica. Este trabajo ha sido posible gracias al proyecto 2014SGR514 (programa "Ajuts a grups de recerca consolidats de la Generalitat de Catalunya").

\section{LITERATURA CITADA}

Anderson, E. F. 2001. The cactus family. Timber Press. Portland, USA. 776 pp.

Arias, S. y E. Sánchez-Martínez. 2010. Una especie nueva de Strombocactus (Cactaceae) del río Moctezuma, Querétaro, México. Rev. Mex. Biodiv. 81: 619-624.

Arzate, K. M. 2009. Distribución de cinco especies de Agave y su relación con algunos parámetros ambientales en Metztitlán, Hidalgo. Tesis de maestría en Ciencias Biológicas. Universidad Nacional Autónoma de México. México, D.F., México. 92 pp. 
López-Pujol et al.: Agave difformis, endemismo mexicano fuera de su área de distribución nativa

Aymerich, P. 2015. Nuevos datos sobre plantas suculentas alóctonas en Cataluña. Bouteloua 22: 99-116.

Badano, E. I. y F. I. Pugnaire. 2004. Invasion of Agave species (Agavaceae) in south-east Spain: invader demographic parameters and impacts on native species. Divers. Distrib. 10: 493-500.

Berger, A. 1915. Die Agaven. Verlag von Gustav Fischer. Jena, Germany. 288 pp.

Boreau, A. 1850. Notice sur l'Agave americana, suivie de la description de quelques plantes nouvelles ou peu connues. Bull. Soc. Ind. Angers Dep. Maine-et-Loire. 21: 249-272.

Bravo-Hollis, H. 1978. Las cactáceas de México. Vol. I. Universidad Nacional Autónoma de México. México, D.F., México. 743 pp.

Britton, N. L. y J. N. Rose. 1919. The Cactaceae. Descriptions and illustrations of plants of the cactus family. Vol. 1. The Carnegie Institution of Washington. Washington, USA. $236 \mathrm{pp}$.

Callen, E. O. 1965. Food habits of some pre-Columbian Mexican Indians. Econ. Bot. 19: 335-343.

Earle, W. H. 1980. Cacti of the Southwest. Rancho Arroyo Book Distributor. Phoenix, USA. $210 \mathrm{pp}$.

Eguiarte, L. E., E. Aguirre-Planter, X. Aguirre, R. Colín, A. González, M. Rocha, E. Scheinvar, L. Trejo y V. Souza. 2013. From isozymes to genomics: Population genetics and conservation of Agave in México. Bot. Rev. 79: 483-506.

Gentry, H. S. 1982. Agaves of continental North America. The University of Arizona Press. Tucson, USA. 670 pp.

Good-Avila, S. V., V. Souza, B. S. Gaut y L. E. Eguiarte. 2006. Timing and rate of speciation in Agave (Agavaceae). Proc. Natl. Acad. Sci. U.S.A. 103: 9124-9129.

Guillot, D. 2006. Un nuevo taxón invasor para la flora balear: Agave ingens A. Berger var. picta (Salm-Dyck) Berger. Blancoana 23: 113-119.

Guillot, D. y P. van der Meer. 2003a. Las familias Agavaceae y Aloaceae en la Comunidad Valenciana. Flora Montiber. 23: 29-43.

Guillot, D. y P. van der Meer. 2003b. Acerca de Agave americana L. var. marginata Trel. en la costa occidental de la Península Ibérica. Toll Negre 2: 16-17.

Guillot, D. y P. van der Meer. 2003c. Acerca de Agave ingens Berger en la Comunidad Valenciana. Toll Negre 2: 18.

Guillot, D. y P. van der Meer. 2004a. Agave $\times$ cavanillesii, nuevo híbrido descubierto en la Comunidad Valenciana. Flora Montiber. 28: 73-76.

Guillot, D. y P. van der Meer. 2004b. Dos nuevos taxones del género Agave descritos en el Jardín Botánico de Valencia. Flora Montiber. 27: 54-56.

Guillot, D. y P. van der Meer. 2004c. Respecto del primer icono del género Agave L. en Europa. Lagascalia 24: 7-17.

Guillot, D. y P. van der Meer. 2005. Nuevos datos de las familias Agavaceae y Aloaceae en la costa mediterránea de la Península Ibérica. Flora Montiber. 30: 3-8.

Guillot, D. y P. van der Meer. 2006a. Claves de las especies del género Agave L. cultivadas como ornamentales en la Península Ibérica e Islas Baleares. Acta Bot. Barc. 50: 441-457.

Guillot, D. y P. van der Meer. 2006b. Un nuevo taxón alóctono potencialmente invasor para la flora ibérica, Agave sisalana var. armata Trel. Stud. Bot. 25: 139-141. 
Guillot, D. y P. van der Meer. 2008. Algunas citas nuevas del género Agave L., para la flora alóctona española. Bouteloua 4: 23-31.

Guillot, D. y P. van der Meer. 2009. Agave desmetiana Jacobi, un nuevo taxón alóctono para la flora europea. Acta Bot. Malacit. 34: 251-254.

Guillot, D. y P. van der Meer. 2010. Nuevos taxones vegetales alóctonos de jardinería en el área continental de NE de España: comportamiento e historia. Manag. Biol. Invasions 1: 6-12.

Guillot, D. y P. van der Meer. 2011. Tres especies del género Agave L. recientemente introducidas en cultivo en España. Bouteloua 8: 8-12.

Guillot, D. y P. van der Meer. 2013a. Agave $\times$ winteriana Berger, un nuevo cultivar en la flora alóctona de la Península Ibérica. Butll. Inst. Catalana Hist. Nat. 77: 153-154.

Guillot, D. y P. van der Meer. 2013b. Primeras fotografías de la inflorescencia de la forma verde de Agave ingens A. Berger, y una nueva cita de esta especie para la flora alóctona española. BV News Publ. Cient. 2013: 47-52.

Guillot, D. y L. Sáez. 2014. Algunas citas nuevas de plantas suculentas en Cataluña. Bouteloua 17: 7-15.

Guillot, D., E. Laguna, J. López-Pujol y C. Puche. 2015. Kalanchoe delagoensis 'Morvedre'. Bouteloua 22: 64-75.

Guillot, D., E. Laguna y J. A. Rosselló. 2009a. La familia Crassulaceae en la flora alóctona valenciana (Monografías de la revista Bouteloua, 4). Jolube Consultor y Editor Ambiental. Teruel y Jaca, España. 106 pp.

Guillot, D., E. Laguna y J. A. Rosselló. 2009b. Flora alóctona valenciana: Familia Cactaceae (Monografías de la revista Bouteloua, 5). Jolube Consultor y Editor Ambiental. Teruel y Jaca, España. 148 pp.

Guillot, D., P. van der Meer, E. Laguna y J. A. Rosselló. 2009c. El género Agave L. en la flora alóctona valenciana (Monografías de la revista Bouteloua, 3). Jolube Consultor y Editor Ambiental. Teruel y Jaca, España. 94 pp.

Invasive Species Compendium. 2015. Kalanchoe delagoensis (chandelier plant) - datasheet. http://www.cabi.org/isc/datasheet/115050 (consultado el 13 de agosto de 2015).

Kornas, J. 1990. Plant invasions in Central Europe: historical and ecological aspects. In: Di Castri, F., A. J. Hansen y M. Debussche (eds.). Biological invasions in Europe and the Mediterranean Basin. Kluwer Academic Publishers. Amsterdam, The Netherlands. pp. 19-36.

Mack, R. N., D. Simberloff, W. M. Lonsdale, H. Evans, M. Clout y F. A. Bazzaz. 2000. Biotic invasions: causes, epidemiology, global consequences, and control. Ecol. Appl. 10: 689-710.

Nyffeler, R. 2003. Aeonium. In: Eggli, U. (ed.). Illustrated handbook of succulent plants: Crassulaceae. Springer. Heidelberg, Germany. pp. 15-23.

Ramón-Laca, L. 1999. Las plantas americanas en la obra de Charles de l'Écluse: primeras citas en las cartas de Juan de Castañeda. An. Jard. Bot. Madr. 57: 97-107.

Rocha, M. 2006. Ecología evolutiva comparada en cinco especies de Agave. Tesis de doctorado. Universidad Nacional Autónoma de México. México, D.F., México. 165 pp.

Rocha, M., A. Valera y L. E. Eguiarte. 2005. Reproductive ecology of five sympatric Agave littaea (Agavaceae) species in Central Mexico. Am. J. Bot. 92: 1330-1341. 
Rubal, J. J., D. Guillot y P. van der Meer. 2013. Dos nuevas especies del género Agave en la provincia de Cádiz (Andalucía, España). Bouteloua 16: 36-39.

Sáez, L., D. Guillot y P. van der Meer. 2014. Nuevas citas de Agaváceas (géneros Agave L. y Yucca L.) en la costa oriental de la Península Ibérica. Bouteloua 18: 131-140.

Sánchez, I., J. García de Lomas y E. Dana. 2009. Aportaciones al conocimiento de la xenoflora gaditana. Lagascalia 29: 296-313.

Sanz, M., E. D. Dana y E. Sobrino. 2004. Atlas de las plantas alóctonas invasoras en España. Dirección General para la Biodiversidad (Ministerio de Medio Ambiente). Madrid, España. 384 pp.

Sanz, M., D. Guillot y V. del Toro. 2011. La flora alóctona de la Comunidad Valenciana (España). Bot. Complut. 35: 97-130.

Scheinvar, E. 2008. Agave difformis Berger. In: Eguiarte, L. y E. Scheinvar (comp.). Agaves y cactáceas de Metztitlán: ecología, evolución y conservación. Universidad Nacional Autónoma de México. México, D.F, México. pp. 44-47.

Serra, L. 2007. Estudio crítico de la flora vascular de la provincia de Alicante: aspectos nomenclaturales, biogeográficos y de conservación. Monografías del Real Jardín Botánico: Ruizia, 19. CSIC (Consejo Superior de Investigaciones Científicas). Madrid, España. 1414 pp.

Smith, G. F. y E. Figueiredo. 2007. Naturalized species of Agave L. (Agavaceae) on the southeastern coast of Portugal. Haseltonia 13: 52-60.

Standley, P. C. 1920. Trees and shrubs of Mexico (Gleicheniaceae-Betulaceae). Contr. U.S. Natl. Herb. 23(1): 1-169.

Thiede, J. 2001. Agavaceae. In: Eggli, U. (ed.). Illustrated handbook of succulent plants: Monocotyledons. Springer. Heidelberg, Germany. pp. 5-102.

Trejo-Salazar, R. E., E. Scheinvar y L. E. Eguiarte. 2015. ¿Quién poliniza realmente los agaves? Diversidad de visitantes florales en 3 especies de Agave (Agavoideae: Asparagaceae). Rev. Mex. Biodiv. 86: 358-369.

Zepeda-Martínez, V., M. C. Mandujano, F. J. Mandujano y J. K. Golubov. 2013. What can the demography of Astrophytum ornatum tell us of its endangered status? J. Arid Environ. 88: 244-249. 